\title{
Improved Sequencing Heuristic DSDV Protocol Using Nomadic Mobility Model for FANETS
}

\author{
Inam Ullah Khan ${ }^{1}$, Muhammad Abul Hassan ${ }^{2}$, Muhammad Fayaz ${ }^{3}$, Jeonghwan Gwak ${ }^{4,5,6,7, *}$ and \\ Muhammad Adnan Aziz
}

\author{
${ }^{1}$ Department of Electronic Engineering, Isra University (SEAS), Islamabad, 44000, Pakistan \\ ${ }^{2}$ Department of Computing and Technology, Abasyn University Peshawar, 25000, Pakistan \\ ${ }^{3}$ Deparment of Computer Engineering, Jeju National University, Jeju, Korea \\ ${ }^{4}$ Department of Software, Korea National University of Transportation, Chungju, 27469, Korea \\ ${ }^{5}$ Department of Biomedical Engineering, Korea National University of Transportation, Chungju, 27469, Korea \\ ${ }^{6}$ Department of AI Robotics Engineering, Korea National University of Transportation, Chungju, 27469, Korea \\ ${ }^{7}$ Department of IT \& Energy Convergence (BK21 FOUR), Korea National University of Transportation, Chungju, 27469, \\ Korea \\ *Corresponding Author: Jeonghwan Gwak. Email: jgwak@ut.ac.kr \\ Received: 04 June 2021; Accepted: 05 July 2021
}

\begin{abstract}
Most interesting area is the growing demand of flying-IoT mergers with smart cities. However, aerial vehicles, especially unmanned aerial vehicles (UAVs), have limited capabilities for maintaining node energy efficiency. In order to communicate effectively, IoT is a key element for smart cities. While improving network performance, routing protocols can be deployed in flying-IoT to improve latency, packet drop rate, packet delivery, power utilization, and average-end-to-end delay. Furthermore, in literature, proposed techniques are very much complex which cannot be easily implemented in realworld applications. This issue leads to the development of lightweight energyefficient routing in flying-IoT networks. This paper addresses the energy conservation problem in flying-IoT. This paper presents a novel approach for the internet of flying vehicles using DSDV routing. ISH-DSDV gives the notion of bellman-ford algorithm consisting of routing updates, information broadcasting, and stale method. DSDV shows optimal results in comparison with other contemporary routing protocols. Nomadic mobility model is utilized in the scenario of flying networks to check the performance of routing protocols.
\end{abstract}

Keywords: Flying-IoT; DSR; smart cities; UAV's; FANETS

\section{Introduction}

A new paradigm of the Smart City model is correlated with quality of life, public security, emergency relief and other urban development resources [1]. Connectivity of aerial vehicles can only be made possible by using internet of things to reshape the concept of smart cities [2]. Due to aerial vehicles changing topological structure, measuring signal strength either indoor or open-air is a tough task. However, proposed model utilized decision tree to find out UAV's location and 
improve signal power [3]. Flying vehicles may be scheduled for effective transfer of items using intelligent transportation. UAVs move in three dimensions which can be useful in agriculture, transportation, \& military missions. As a result, the urgent demand for vehicle-aided computing in aerial vehicles for smart cities is introduced [4]. In general, Fig. 1 demonstrates the concept of smart cities integration with aerial vehicles.

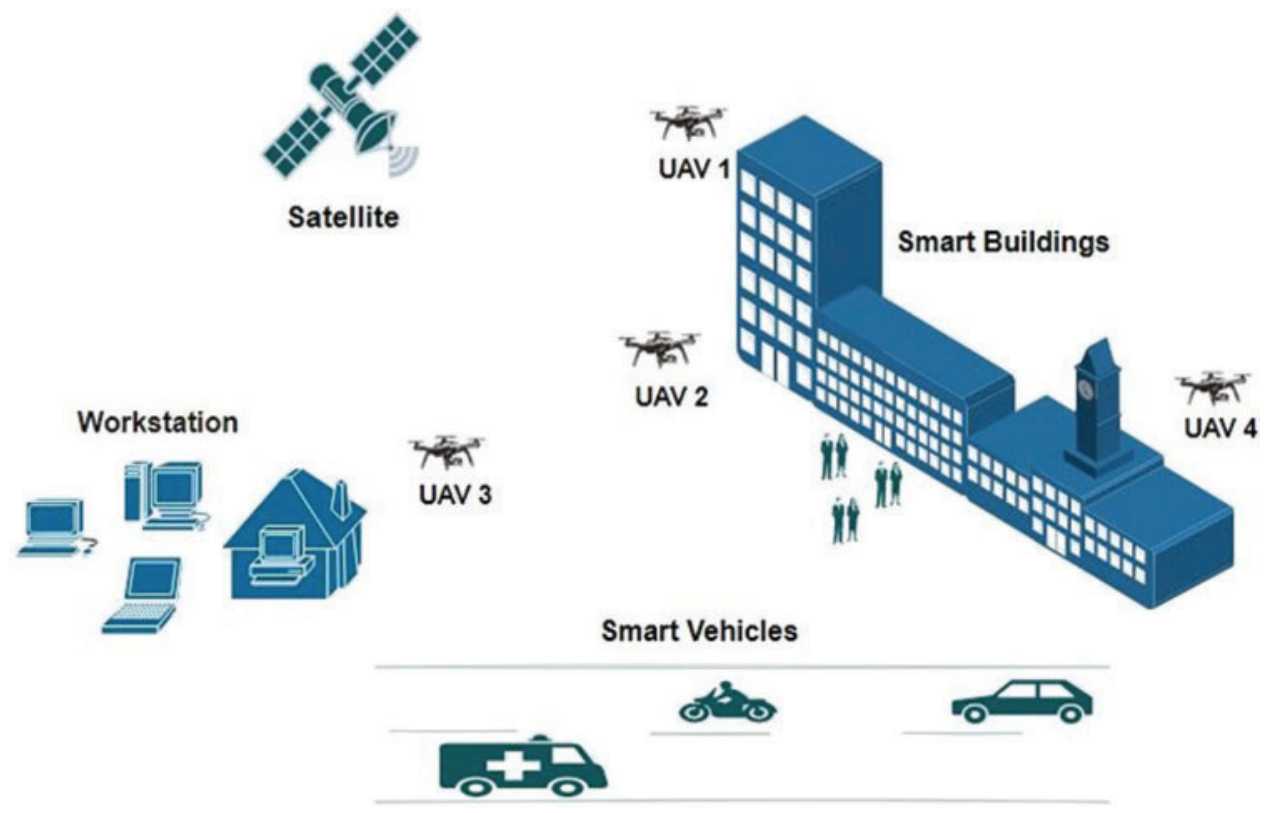

Figure 1: Smart cities integration with flying-IoT network

Aerial ad hoc networks require routing protocols for selecting optimal route selection. Enhance-AntHocNet routing technique is suggested to increase network lifetime. A novel parameter energy stabilizing threshold is incorporated in working of ant colony optimization to conserve UAV's energy [5]. Flying ad hoc network (FANET) has an advantage over other areas, due to its availability, easy and fast deployment in every situation. It also enables inter- and intra-UAV networking for collaboration and cooperation among the Internet of Flying Things (IoFT) [6]. Moreover, multi-point relay (MPR) technique can be utilized to tackle duplicate data transmission of packets illustrated in Fig. 2 Since UAVs operate in swarms and exchange data in offline mode, they can compile data without access to the internet. FANETs have enhanced IoFT with no Line of Sight (LoS) issues and data sharing from very far missions. UAVs are lightweight and can move in any direction at a speed of $30-460 \mathrm{~km} / \mathrm{h}$. Due to changing network topology frequently during operation data routing from air-to-air and air-to-ground suffers. The ability to embed an effective routing protocol is a key component in the life of UAVs. Drones are categorized into two types: F-i-x-e-d- W-i-n-g (FW) and R-o-t-a-r-y-W-i-n-g (RW). Fixed-wing aerial vehicles are more capable for longer surveillance fight time with optimal aero-dynamic design and flight speed. In contrast, rotary-Wing can vertically take-off from any position and fly in much lower altitudes [7]. Tab. 1 describes the main difference between Fixed-wing and Rotary-wing. 


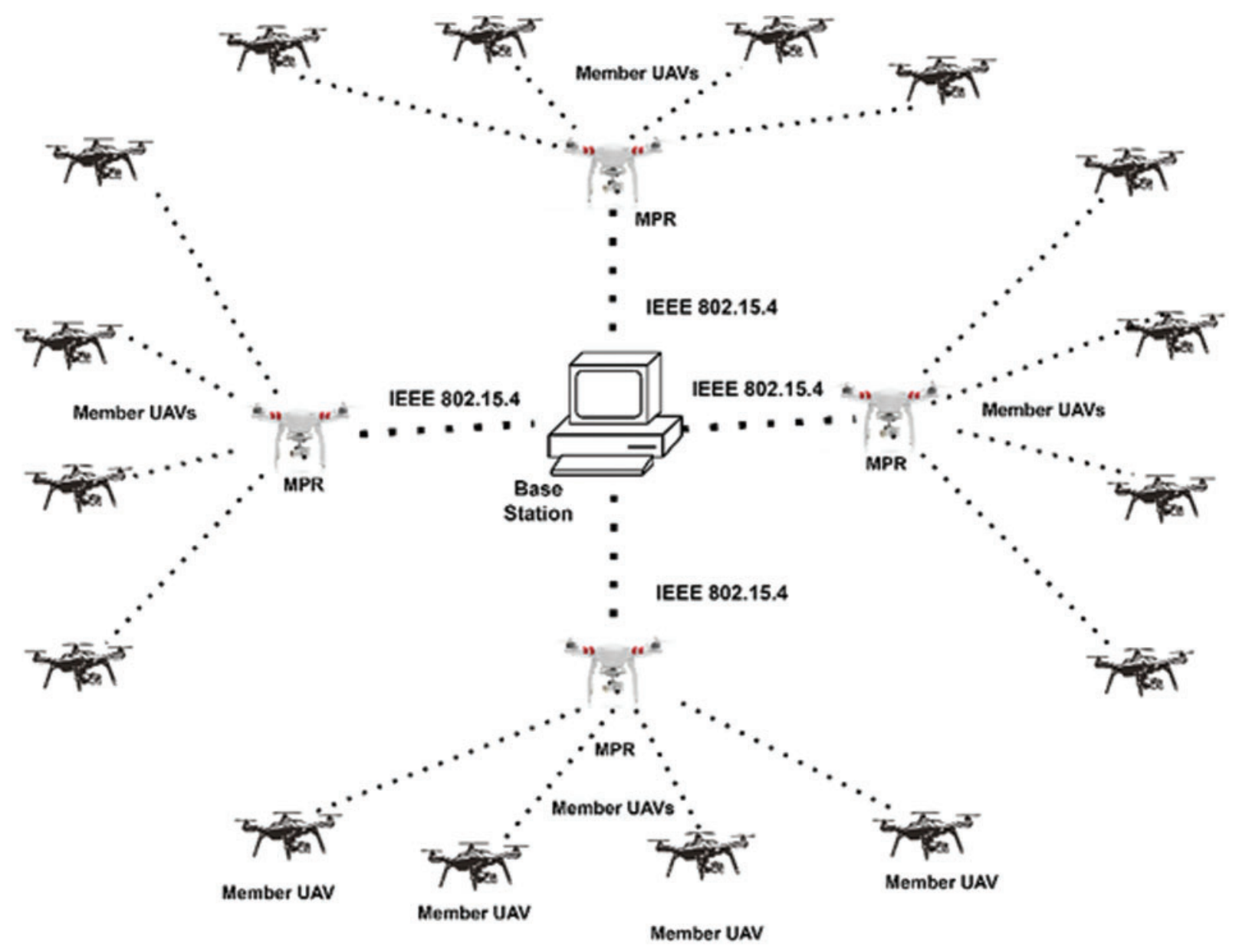

Figure 2: Multi-point relay

Table 1: Fixed and rotary UAVs having metrics energy, static \& altitude

\begin{tabular}{llllll}
\hline Size of UAV's & Types & Energy & Mobility & Static & Altitude \\
\hline Large size UAV & FW & High & Low & No & High \\
& RW & Low-M & M-H & Yes & M-H \\
Small size UAV & FW & M-H & M-H & No & L-M \\
& RW & Low & Low & Yes & Low \\
\hline
\end{tabular}

Intelligent UAVs are small in size and light-weighted with limited resources, such as bandwidth and storage. In the light of the above problems, the contributions of this article are as below.

- Implementation of various routing protocols in the area of flying Ad-hoc networks.

- Novel routing scheme ISH-DSDV is introduced in aerial networks.

- Protocols are investigated using metrics which are jitter, throughput, channel/bandwidth utilization, packet drop rate, packet loss, packet delivery ratio.

- Nomadic mobility model is used to enhance the capabilities of UAV's. 


\section{Literature Review}

The diverse nature of IoT devices gives solutions to design smart cities. In smart cities, IoT has allowed various types of devices to communicate and share information using special protocols. It also offers services to citizens and government entities. IoT-based routing protocol is introduced to strengthen various connected gadgets. The best-fit approach reinforces to repair channel links while connecting with nodes. However, the alternative opportunistic routing, which eliminates unnecessary data packets while improving transmission end-to-end delay to boost-up performance [8]. UAVs and fifth-generation (5G) IoT networks were introduced for Smart Cities. Novel hierarchical multi-UAV architecture is proposed for collaboration and trajectory problemsolving. The UAV mission control pattern distribution and swarm behaviors which address to elect cluster head. Among aerial vehicles and base stations, the use of $5 \mathrm{G}$ technologies helps to prevent handover problems [9]. The loss of connection breakage is caused by low-power energy nodes. As a result, an attacker will easily breach the network; thus, a robust neural technique called back propagation learning algorithm is used to protect communication in IoT-based networks [10]. Although intelligent IoT introduces multi-tier computing, which integrates Cloud, Fog, and Edge approaches [11]. However, attackers can access a complex IoT network by increasing the length of transmitted packets in order to degrade resources. An intrusion detection system is programmed to filter data packets that cause disruption [12]. Decision-making is having a special role in between aerial vehicles and IoT devices while transferring data packets. In order to mitigate packet length and balancing node energy, the Markov decision mechanism formulates a threshold that allows UAVs to transfer energy. UAV-enabled wireless power transfer system shows the dependency of each node to solve cognitive processes [13]. At the same time, aerial vehicles take part in industrial internet of things (IIoT) applications for inter and intra communication to perform optimal multipath routing [14]. A hybrid algorithm is proposed which is based on Markov decision process and random serial dictatorship for wireless charging of IoT devices using unmanned aerial vehicles [15].

Scientists have observed a number of problems with flying vehicles, including congestion, delay, and unbalanced energy while receiving data packets. While delivering value-added internet of things services to maintain a trade-off between energy consumption and operating time, although providing a solution to delay and energy-aware UAV selection [16]. AntHocNet routing is the approach inspired from the behavior of ants which can be implemented with different mobility models in the area of flying networks. Using our proposed scheme, ISH-DSDV is a table-driven routing by targeting flying ad hoc networks. To control the problem of energy imbalance and conserve aerial vehicles network life-time ISH-DSDV routing is formulated.

\section{Proposed Scheme}

The DSDV routing protocol is used in the dynamics of aerial networks in this section. In addition, each UAV receives routing packet information from flying networks, which causes topology changes or, in some cases, forwards data packets and terminates the process. Because of the deployment of DSDV, every aerial node must have all of neighbors' information. To avoid congestion, this strategy uses a unique mechanism called sequence number allocation by target node, which optimizes phase shifts. Every flying-IoT node prefers high sequence number in comparison with low. This protocol updates information in the routing table, which includes three metrics: final destination, distance, and next node [17]. Formulated approach gives the best use of information during transferring from one node to another. Working in the area of flying networks, a stale data entry method is introduced, which allows for the easy removal of entries that are 
not updated. Whereas the Fig. 3, elaborates the concept of improved sequencing heuristic based DSDV routing protocol for flying networks. The proposed algorithm consists of the following steps which are as under.

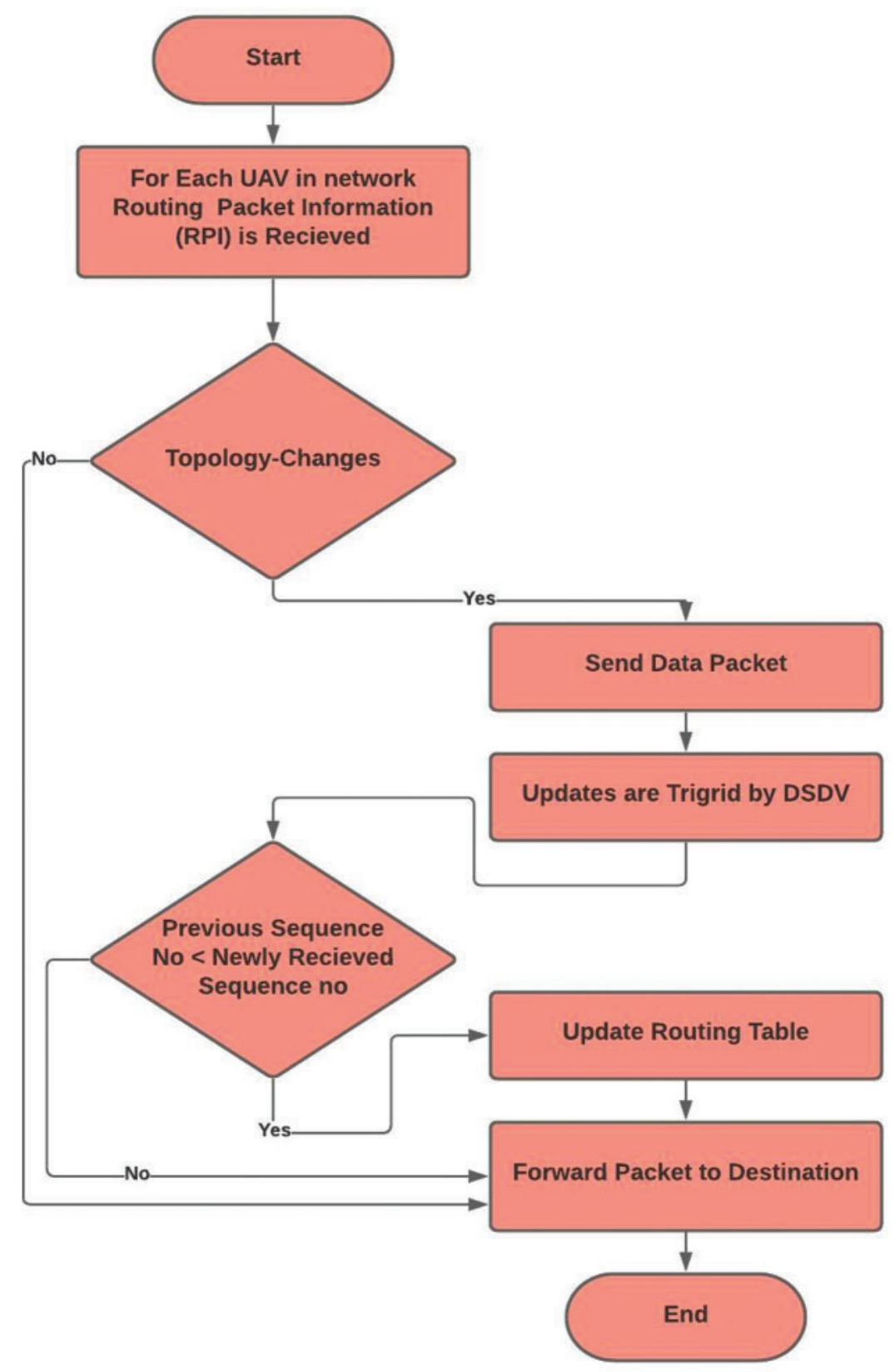

Figure 3: Improved sequencing heuristic DSDV routing protocol

- Incremental Updates work.

This parameter updates the overall routing table and broadcasts to all nodes in the net- 
- Full-Dumps

Offers the best loop-free routing method.

- Stale-data process

The UAV will be automatically removed from the network if the flying node does not refresh the routing table information.

The ISH-DSDV is the optimum technique for the internet of flying vehicles because it is appropriate for a minimal number of nodes. This method, on the other hand, is ideal for dynamic pattern-based flying networks.

\section{Mobility Model}

Using the idea of nomadic mobility in this research, flying nodes move randomly at a specific point. Within the zone, aerial vehicles cover the maximum distance from the reference point. A random way point is employed while going from one region to another. This mobility pattern divides the flying-space to avoid collision [18]. Fig. 4 shows the moment of thirty unmanned aerial vehicles using nomadic mobility model.

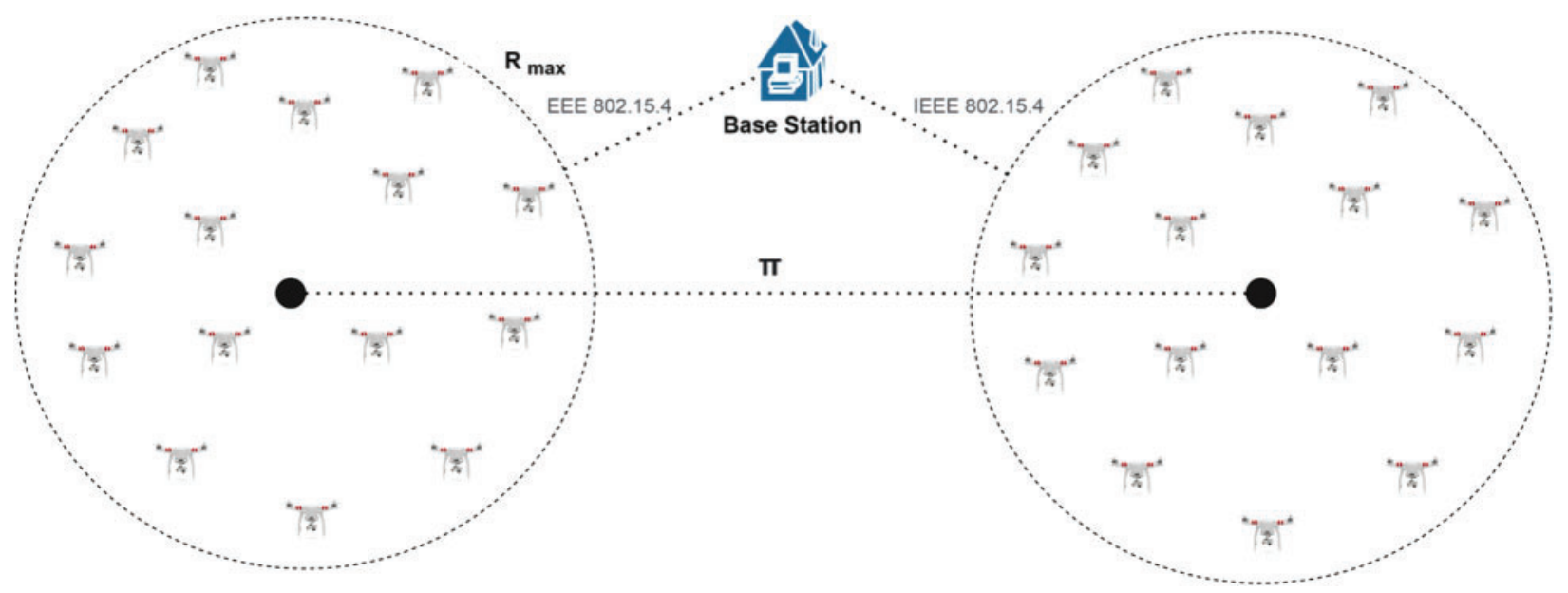

Figure 4: Network topology of UAV-network using nomadic mobility model

\section{Simulation Environment Setup}

UAV's are placed in a diverse topological structure having network size of $1000 \mathrm{~m} \times 1000 \mathrm{~m}$. The flying network employs a nomadic mobility paradigm with thirty-one nodes, one of which is a land station. For comparison, we examined six routing protocols, and our proposed solution is based on DSDV routing.

\section{Simulation Metrics}

This section includes the parameters to evaluate the behavior of the network.

\subsection{Network Throughput}

The rate of successful packets delivered over a communication medium to the final destination. Throughput is measured in kilobyte per second (Kbps), as shown in Eq. (1). Internet 
of flying vehicles faces a lot of problems due to dynamic behavior. In terms of throughput estimation, moreover, AntHocNet and DSDV routing shows better results. AntHocNet, AOMDV, DSDV, DSR, M-DART and ZRP routing methods are used to evaluate network throughput in Tab. 2 and Fig. 5. While the graphical view of Tab. 2 is mentioned in Fig. 6.

Throughput $=\sum\left(\frac{\text { received packetsize }}{\text { Time }}\right)($ Kbps $)$

Table 2: Throughput analysis of nomadic mobility model (Kbps)

\begin{tabular}{lllllll}
\hline Protocols & AntHocNet & AOMDV & DSDV & DSR & M-DART & ZRP \\
\hline Minimum & 40.15625 & 77.5625 & 24 & 60 & 24 & 60.96875 \\
Maximum & 509.8125 & 717.3125 & 742.25 & 712 & 489.0313 & 655.4375 \\
Average & 289.864986 & 434.7788 & 544.2037 & 423.4696 & 162.3531 & 496.9663 \\
Standard deviation & 104.887032 & 177.3229 & 171.5673 & 207.6131 & 104.4426 & 100.2816 \\
\hline
\end{tabular}

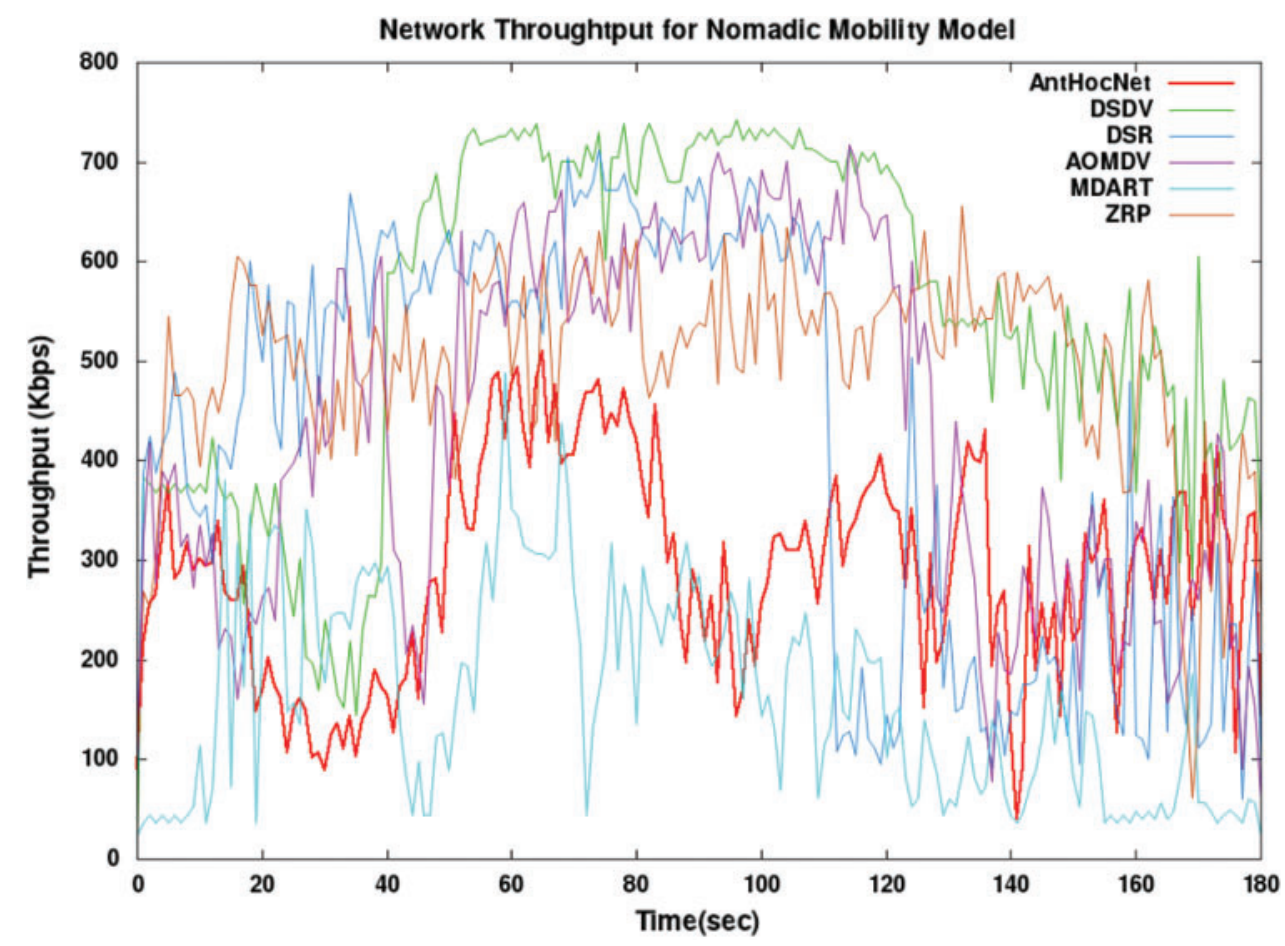

Figure 5: Network throughput for nomadic mobility model

\subsection{Packet Drop Analysis}

Number of data packets drops, it causes delays and has a negative effect on the performance of the service. High level of latency indicates a less reliable network which is not feasible for fastchanging topological networks. Tab. 3 describe packet drop rate study of each routing protocol. Therefore the packet drop counts are presented in Figs. $7 \& 8$ which shows the abstract graphical view. 


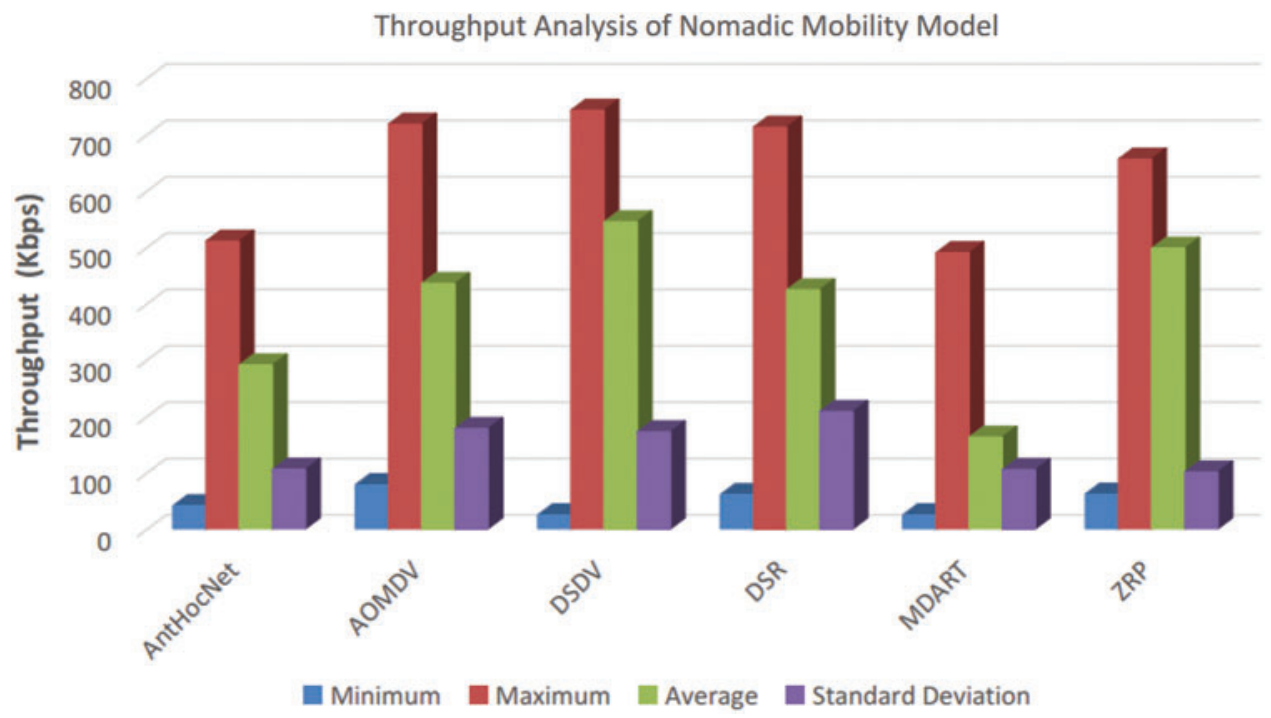

Figure 6: Throughput analysis of different protocols using nomadic mobility model

Table 3: Packet drop rate for nomadic mobility model (Kbps)

\begin{tabular}{lllllll}
\hline Protocols & AntHocNet & AOMDV & DSDV & DSR & M-DART & ZRP \\
\hline Minimum & 1 & 2 & 49 & 3 & 30 & 36 \\
Maximum & 1480 & 918 & 300 & 381 & 718 & 158 \\
Average & 135.444444 & 187.9444 & 171.5083 & 187.7459 & 2629779 & 96.30409 \\
Standard deviation & 239.250687 & 174.4598 & 46.81415 & 70.9609 & 75.31371 & 23.63145 \\
Total & 24380 & 33830 & 30946 & 33828 & 47428 & 16468 \\
\hline
\end{tabular}

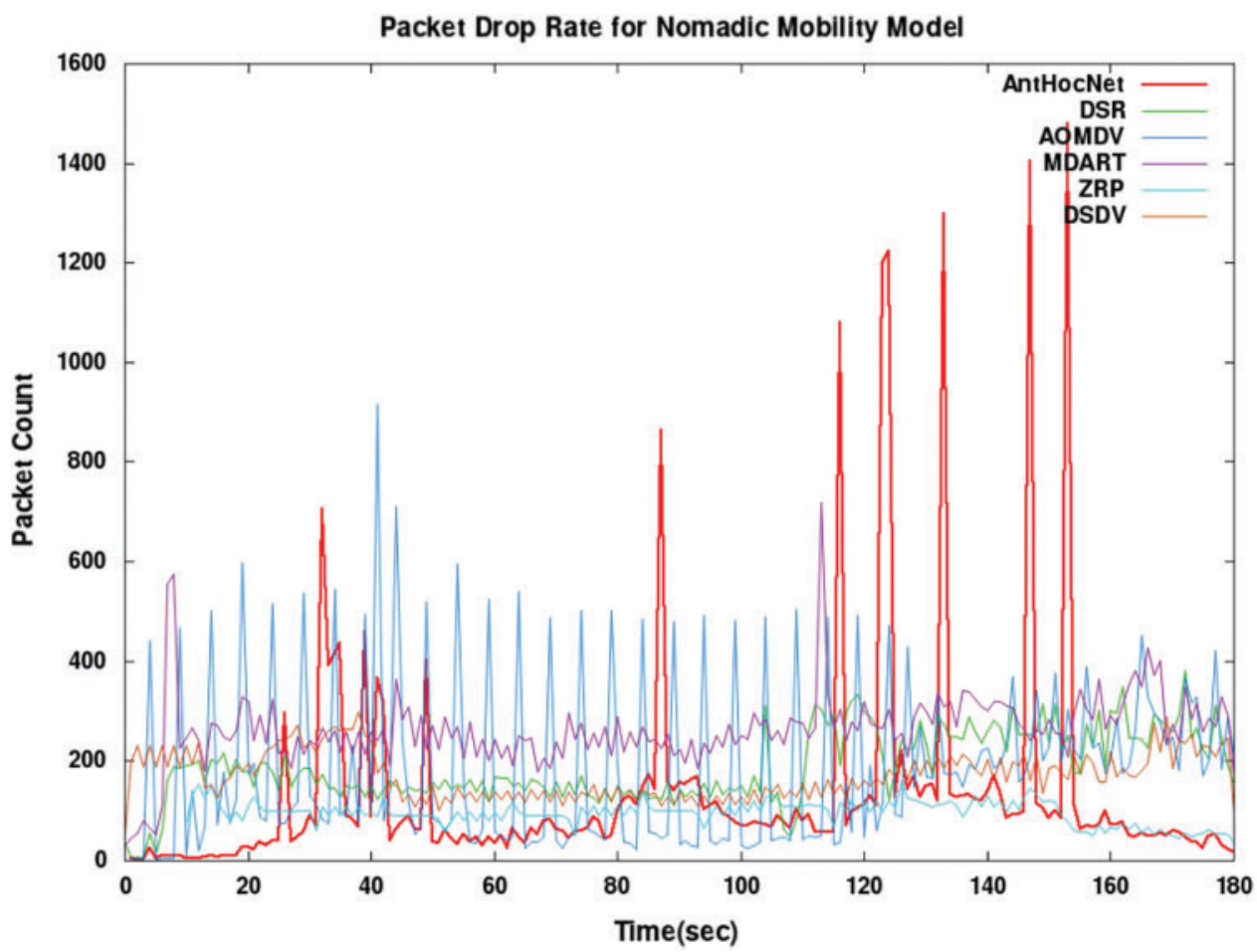

Figure 7: Packet drop rate for nomadic mobility model 


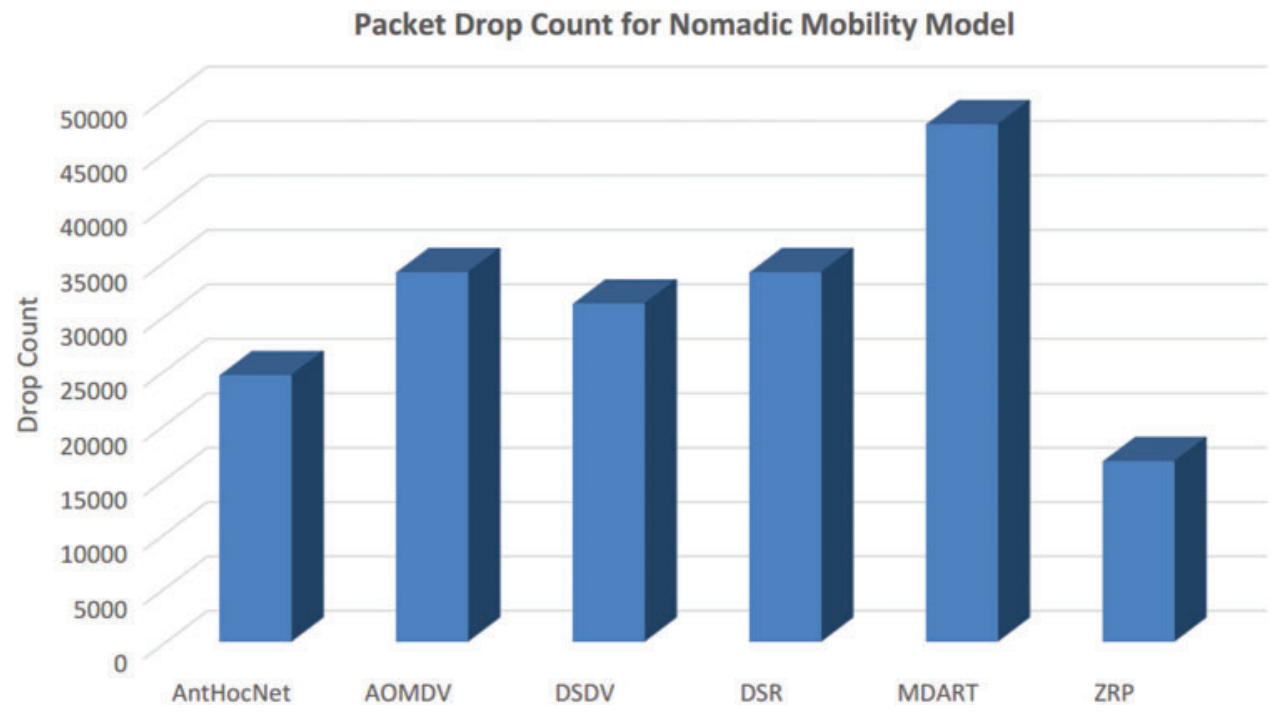

Figure 8: Packet drop count using six routing protocols

\subsection{Packet Delivery Ratio}

Total packets delivered by every-node in per unit time. In this research study, the packet delivery ratio is derived from the following formula. DSDV provides optimal analysis in the area of internet of flying networks by enhancing packet delivery ratio. Fig. 9 shows three parameters: data packets sent and received data packets. Tab. 4 depicts the routing approaches used to compare by applying PDR.

Packet Delivery ratio $=100 * \frac{\text { Packet Delivered }}{\text { Total Number of Packets }}$

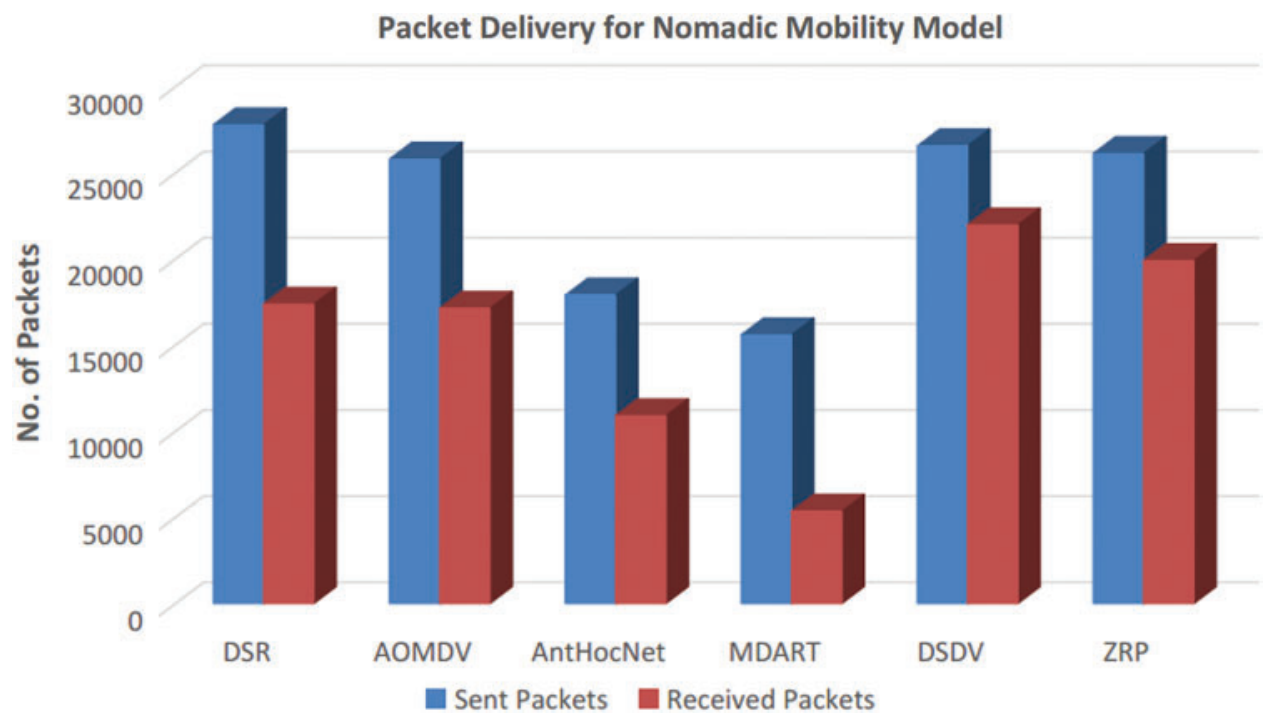

Figure 9: Packet delivery ratio using nomadic mobility model for UAV-network 
Table 4: Packet delivery ratio (PDR)

\begin{tabular}{llll}
\hline Protocols & Sent packets & Received packets & PDR (\%) \\
\hline DSR & 27775 & 17361 & 62.50585 \\
AOMDV & 25776 & 17111 & 66.38346 \\
AntHocNet & 17884 & 10890 & 60.89242 \\
M-DART & 15582 & 5337 & 34.25106 \\
DSDV & 26547 & 21965 & 82.74005 \\
ZRP & 26109 & 19888 & 76.17297 \\
\hline
\end{tabular}

\subsection{Packet Loss}

The parameter packet-loss is well explained in Tab. 5 and Fig. 10 shows the same metrics in graphical form where different routing protocols are used. DSDV shows very less packet loss in comparison with other routing techniques. However Tab. 5 is having the brief study regarding packet loss using DSR, AOMDV, AntHocNet, M-DART, DSDV \& ZRP.

Table 5: Packet loss

\begin{tabular}{llll}
\hline Protocols & Sent packets & Received packets & Packet loss \\
\hline DSR & 27775 & 17361 & 10414 \\
AOMDV & 25776 & 17111 & 8665 \\
AntHocNet & 17884 & 10890 & 6994 \\
M-DART & 15582 & 5337 & 10245 \\
DSDV & 26547 & 21965 & 4582 \\
ZRP & 26109 & 19888 & 6221 \\
\hline
\end{tabular}

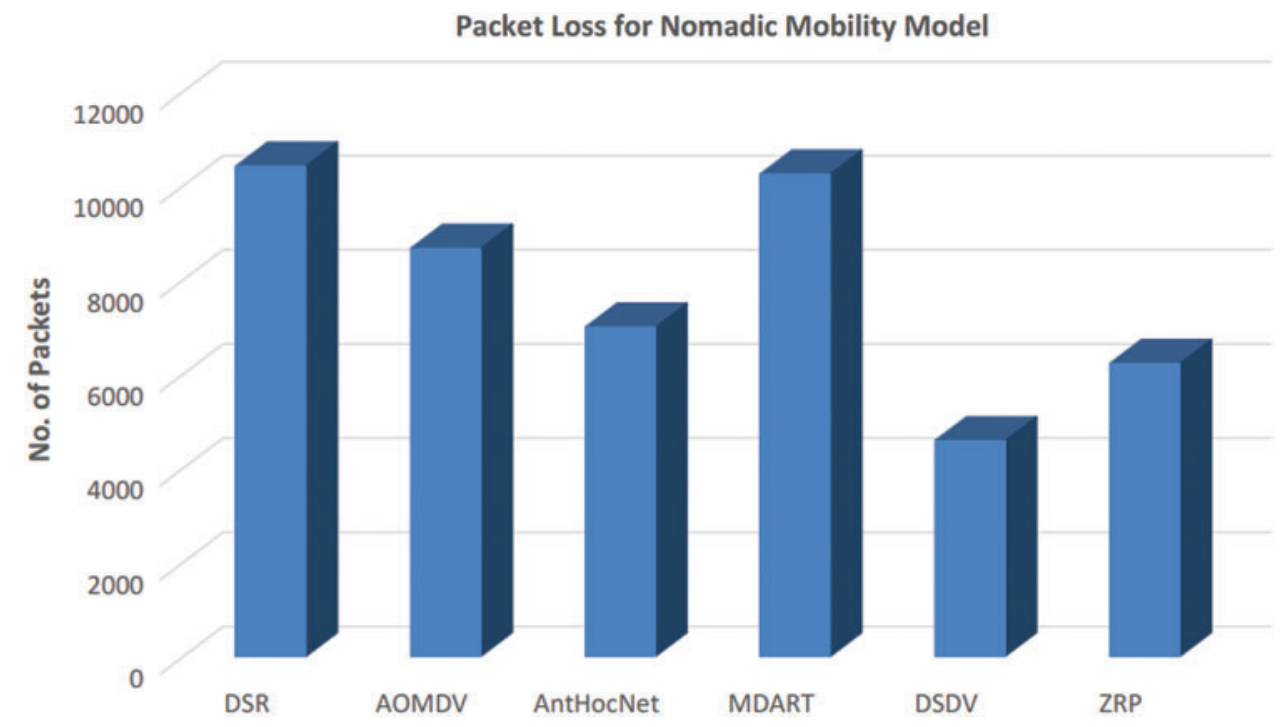

Figure 10: Packet loss 


\subsection{Bandwidth/Channel Utilization}

The overall sum of the delivered data packets divided by per unit time in kilo bits per second use to calculate network utilization. Channel utilization with two different scenarios in flying-IoT networks are given in Tab. 6. The performance is properly checked and presented in Fig. 11.

Table 6: Network utilization for nomadic mobility model (Kbps)

\begin{tabular}{lllllll}
\hline Protocols & AntHocNet & AOMDV & DSDV & DSR & M-DART & ZRP \\
\hline Minimum & 893.5 & 1000.578 & 848.5938 & 1502.656 & 720 & 720 \\
Maximum & 8895.4375 & 3965.969 & 3623.031 & 5241.734 & 3956.547 & 1320 \\
Average & 8895.4375 & 3965.969 & 3623.031 & 5241.734 & 3956.547 & 1320 \\
Standard Deviation & 1154.10007 & 426.9384 & 408.6488 & 692.3719 & 500.1647 & 129.4262 \\
\hline
\end{tabular}

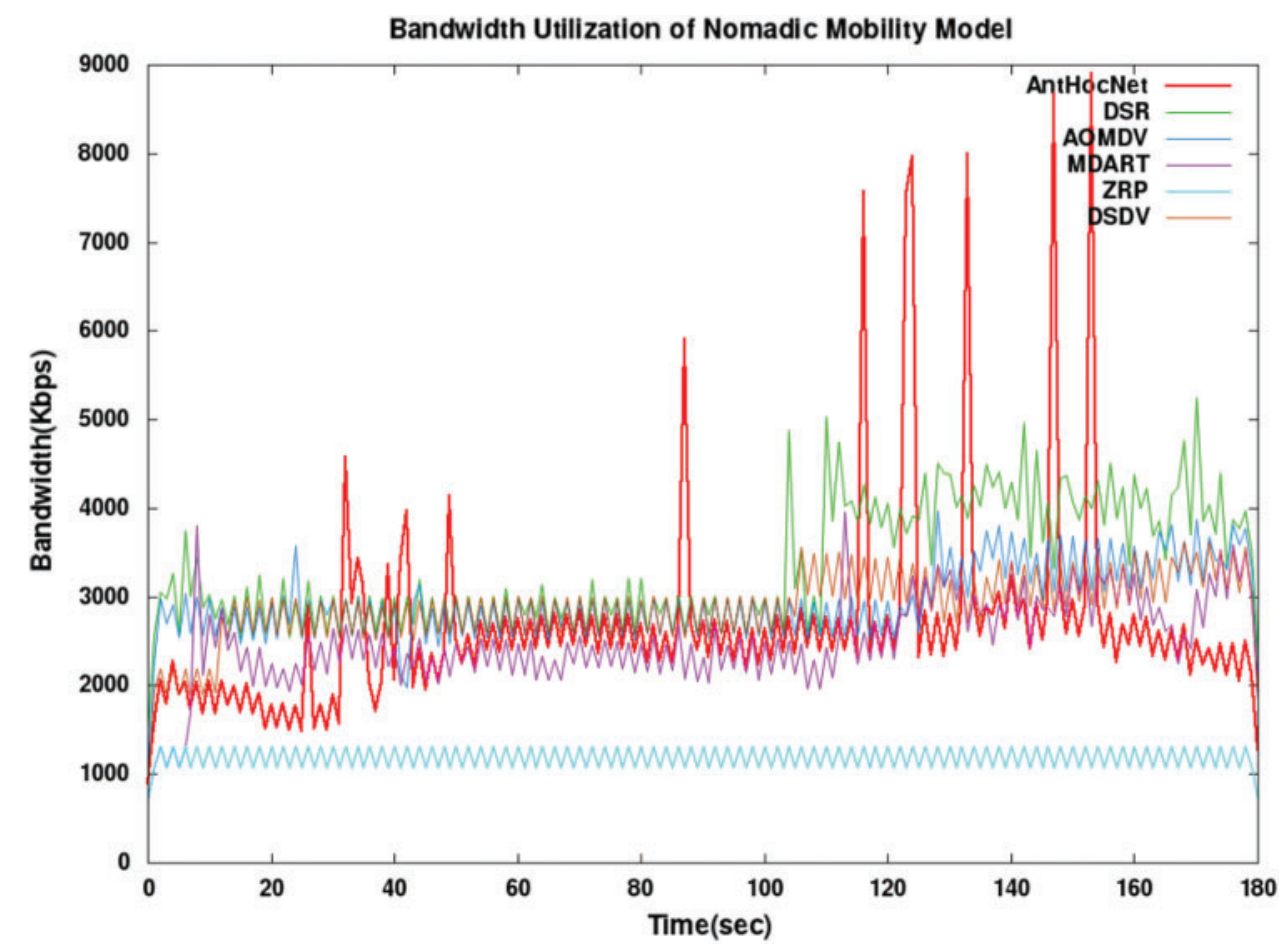

Figure 11: Bandwidth/Channel utilization using nomadic mobility model

\subsection{Average End-to-End Delay}

Network flow in NS-2(v2.35) may trace the cycle from initializing information packets until it reach by the target node. While doing simulation three routing techniques shows better results which include DSDV, M-DART and ZRP. Fig. 12 and Tab. 7 illustrates DSDV routing is having 
very less delay factor in comparison with other protocols. Eq. (3) shows delay formula which are as under.

Average End-to-End Delay $=\mid$ Receive Time $a v g|-|$ Send Time ${ }_{\text {avg }} \mid$

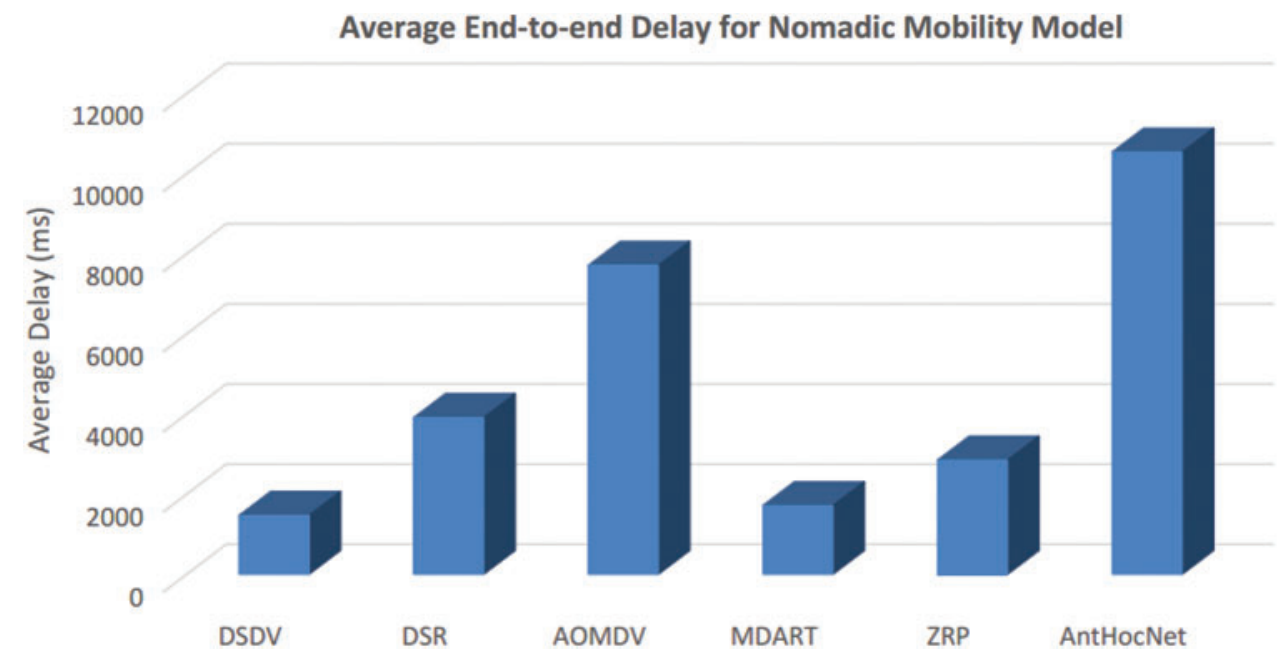

Figure 12: Average-end-to-end delay using nomadic mobility model

Table 7: Average end-to-end-delay for nomadic mobility model

\begin{tabular}{ll}
\hline Protocols & Average end-to-end-delay (ms) \\
\hline DSDV & 1468 \\
DSR & 3908 \\
AOMDV & 7707 \\
M-DART & 1714 \\
ZRP & 2849 \\
AntHocNet & 10538 \\
\hline
\end{tabular}

\section{Conclusion \& Future Directions}

Flying-IoT with the integration of smart cities is the better approach to overcome on end-toend delay. FANETs are the most reliable and easy to deploy in any situation, which has gained the interest of the scientific community and industrial stakeholders. Flying-IoT works mostly in three-dimensional environments to better support applications. The key problem that a flying-IoT network faces is to ensure that all limited resources can be used effectively. The main aim behind this research study is to address resource problems in terms of throughput, bandwidth, End-toEnd delay, packet delivery ratio. In the area of flying-IoT networks, DSDV is lightly weighted for enhancing network lifetime. The nomadic mobility model has played an essential role in solving the problems associated with UAV networks.

In overall analysis, the proposed solution has performed well and utilized resources more efficiently. Simulation results showed that light-weighted-ISH-DSDV is a better choice to deploy 
into the dense network. In the future, for secure communication in smart cities, cyber-secure protocol will be a novel contribution.

Funding Statement: This work was supported by the Basic Science Research Program through the National Research Foundation of Korea (NRF) funded by the Ministry of Education (Grant No. NRF-2020R1I1A3074141), the Brain Research Program through the NRF funded by the Ministry of Science, ICT and Future Planning (Grant No. NRF-2019M3C7A1020406), and "Regional Innovation Strategy (RIS)" through the NRF funded by the Ministry of Education.

Conflicts of Interest: The authors declare that they have no conflicts of interest to report regarding the present study.

\section{References}

[1] S. H. Alsamhi, O. Ma, M. S. Ansari and F. A. Almalki, "Survey on collaborative smart drones and internet of things for improving smartness of smart cities," IEEE Access, vol. 7, pp. 128125-128152, 2019.

[2] N. Awan, S. Khan, M. K. I. Rahmani, M. Tahir, N. Alam et al., "Machine learning-enabled power scheduling in IoT-based smart cities," Computers, Materials \& Continua, vol. 67, no. 2, pp. 2449-2462, 2021.

[3] I. U. Khan, R. Alturki, H. J. Alyamani, M. A. Ikram, M. A. Aziz et al., "RSSI controlled long range communication in secured IoT-Enabled unmanned aerial vehicles," Mobile Information Systems, vol. 2021, pp. 1-11, 2021.

[4] M. Dai, Z. Su, Q. Xu and N. Zhang, "Vehicle assisted computing offloading for unmanned aerial vehicles in smart city," IEEE Transactions on Intelligent Transportation Systems, vol. 22, no. 3, pp. 19321944, 2021.

[5] I. U. Khan, I. M. Qureshi, M. A. Aziz, T. A. Cheema and S. B. H. Shah, "Smart IoT control-based nature inspired energy efficient routing protocol for flying ad hoc network (FANET)," IEEE Access, vol. 8, pp. 56371-56378, 2020.

[6] D. F. Pigatto, M. Rodrigues, J. V. D. C. Fontes, A. S. R. Pinto, J. Smith et al., "The internet of flying things," Internet of Things A to Z: Technologies and Applications, vol. 165, pp. 529-562, 2018.

[7] A. G. Perez and M. D. Cano, "Flying ad hoc networks: A new domain for network communications," Sensors, vol. 18, no. 10, pp. 3571, 2018.

[8] A. A. AlZubi, M. A. Maitah and A. Alarifi, "A best-fit routing algorithm for non-redundant communication in large-scale IoT based network," Computer Networks, vol. 152, no. 7, pp. 106-113, 2019.

[9] F. Qi, X. Zhu, G. Mang, M. Kadoch and W. Li, "UAV network and IoT in the sky for future smart cities," IEEE Network, vol. 33, no. 2, pp. 96-101, 2019.

[10] N. Chen, T. Qiu, X. Zhou, K. Li and M. Atiquzzaman, "An intelligent robust networking mechanism for the Internet of Things," IEEE Communications Magazine, vol. 57, no. 11, pp. 91-95, 2019.

[11] Y. Yang, "Multi-tier computing networks for intelligent IoT," Nature Electronics, vol. 2, no. 1, pp. 4-5, 2019.

[12] A. Abdollahi and M. Fathi, "An intrusion detection system on ping of death attacks in IoT networks," Wireless Personal Communication, vol. 112, no. 4, pp. 2057-2070, 2020.

[13] S. Lhazmir, O. A. Oualhaj, A. Kobbane and L. Mokdad, "A decision-making analysis in UAV-enabled wireless power transfer for IoT networks," Simulation Modelling Practice and Theory, vol. 103, no. 1, pp. $1-13,2020$.

[14] F. A. Turjman and S. Alturjman, "5G/IoT-enabled UAVs for multimedia delivery in industry-oriented applications," Multimedia Tools and Applications, vol. 79, no. 13, pp. 8627-8648, 2020. 
[15] C. Su, F. Ye, L. C. Wang, L. Wang, Y. Tian et al., "UAV-assisted wireless charging for energyconstrained IoT devices using dynamic matching," IEEE Internet of Things Journal, vol. 7, no. 6, pp. 4789-4800, 2020.

[16] N. H. Motlagh, M. Bagaa and T. Taleb, "Energy and delay aware task assignment mechanism for UAV-based IoT platform," IEEE Internet of Things Journal, vol. 6, no. 4, pp. 6523-6536, 2019.

[17] A. Nayyar, "Flying Adhoc Network (FANETs): Simulation based performance comparison of routing protocols," in 2018 Int. Conf. on Advances in Big Data, Computing and Data Communication Systems, Durban, South Africa, pp. 1-9, 2018.

[18] A. Bujari, C. T. Calafate, J. C. Cano, P. Manzoni, C. E. Palazzi et al., "Flying ad-hoc network application scenarios and mobility models," International Journal of Distributed Sensor Networks, vol. 13, no. 10, pp. 1-17, 2017. 